\title{
THE ASSOCIATION OF PRIMARY ANESTHESIA TYPE WITH POSTOPERATIVE ADVERSE EVENTS FOLLOWING MINIMALLY INVASIVE TRANSCAROTID ARTERY REVASCULARIZATION
}

John J. Finneran IV MD, Brittany N. Burton MHS, MAS, Matthew W. Swisher MD, MS, Jerry Ingrande MD, MS, and Rodney A. Gabriel MD, MS

Department of Anesthesiology, University of California, San Diego

\section{INTRODUCTION}

The anesthesia literature remains sparse regarding influence of primary anesthesia type with adverse events following minimally invasive transcarotid artery revascularization (TCAR). ${ }^{1}$ The objective was to report the association of primary anesthesia type with 30-day adverse events following TCAR.

\section{RESULTS}

Final analysis included 625 patients. The prevalence of regional anesthesia/MAC was $73.4 \%$. We observed a 93\% decrease in the odds of 30-day mortality $(p=0.018)$ in patients who received regional anesthesia/MAC. Mean [SD] hospital stay (2.99 [5.92] days versus 4.30 [9.15] days, $\mathrm{p}=0.037)$ and case duration (88.45 [39.48] minutes versus 105.85 [63.77] minutes, $p<0.001$ ) were shorter among patients who received regional anesthesia/MAC.

Pulmonary complications were lower in the regional/MAC group $(p=0.021)$.

\section{DISCUSSION}

The majority of studies on this topic pertain to carotid endarterectomy patients, this analysis sheds light on outcomes following TCAR. Overall, we urge further risk stratification and pre-procedural optimization to carefully select patients who may undergo regional/MAC.

\section{METHODS}

The American College of Surgeons National Surgical Quality Improvement Program registry from 2012 to 2016 was used for this retrospective study. The primary exposure was anesthesia type (regional/MAC versus general anesthesia). Primary endpoint was 30-day mortality. Secondary 30-day endpoints included pulmonary, renal, and cardiac complications, sepsis, DVT, stroke, transfusion, thrombosis of ipsilateral carotid vessel, reoperation. Logistic regression was used to evaluate the association of anesthesia type with adverse events.

\begin{tabular}{|c|c|c|c|}
\hline & \multicolumn{2}{|c|}{ Primary Anesthesia } & \multirow[b]{2}{*}{$P$ Value } \\
\hline & General Anesthesia & Regional/MAC & \\
\hline Sample No. (\%) & 166 & 459 & \\
\hline Hospital stay (days), mean (SD) & $4.30(9.15)$ & $2.99(5.92)$ & 0.037 \\
\hline Case Duration (minutes), mean (SD) & $105.85(63.77)$ & $88.45(39.48)$ & $<0.001$ \\
\hline Pulmonary Complications & $8(4.8)$ & $6(1.3)$ & 0.021 \\
\hline Pneumonia & $6(3.6)$ & $5(1.1)$ & 0.076 \\
\hline Mechanical Ventilation & $3(1.8)$ & $3(0.7)$ & 0.400 \\
\hline Reintubation & $5(3.0)$ & $5(1.1)$ & 0.183 \\
\hline $\mathrm{PE}$ & $1(0.6)$ & $0(0.0)$ & 0.595 \\
\hline Sepsis Complication & $3(1.8)$ & $1(0.2)$ & 0.103 \\
\hline Sepsis & $2(1.2)$ & $1(0.2)$ & 0.357 \\
\hline Septic Shock & $1(0.6)$ & $0(0.0)$ & 0.595 \\
\hline Renal Complications & $2(1.2)$ & $3(0.7)$ & 0.861 \\
\hline UTI & $1(0.6)$ & $2(0.4)$ & 1.00 \\
\hline Renal Insufficiency & $1(0.6)$ & $0(0.0)$ & 0.595 \\
\hline AKI & $0(0.0)$ & $1(0.2)$ & 1.00 \\
\hline DVT & $3(1.8)$ & $0(0.0)$ & 0.026 \\
\hline CVA & $9(5.4)$ & $15(3.3)$ & 0.316 \\
\hline Cardiac Complications & $2(1.2)$ & $10(2.2)$ & 0.650 \\
\hline $\mathrm{Ml}$ & $1(0.6)$ & $10(2.2)$ & 0.327 \\
\hline CPR & $1(0.6)$ & $0(0.0)$ & 0.595 \\
\hline Infection & $0(0.0)$ & $1(0.2)$ & 1.00 \\
\hline Superficial SSI & $0(0.0)$ & $1(0.2)$ & 1.00 \\
\hline Blood Transfusion & $13(7.8)$ & $15(3.3)$ & 0.027 \\
\hline Embolism/Thrombosis & $4(2.4)$ & $4(0.9)$ & 0.268 \\
\hline Reoperation & $8(4.8)$ & $13(2.8)$ & 0.334 \\
\hline Death & $6(3.6)$ & $3(0.7)$ & 0.018 \\
\hline
\end{tabular}

\section{REFERENCES}

1. Paraskevas KI, de Borst GJ, Eckstein HH, Schermerhorn ML. Transfemoral vs Transcervical Carotid Artery Stenting. J Endovasc Ther. 2019; 26(2): 228-230.

2. American College of Surgeons NSQIPAN. ACS NSQIP Participant Use Data File. 